\title{
An Improved Anonymous Remote user Authentication Scheme with Key Agreement based on Dynamic Identity
}

\author{
Yajuan Shi ${ }^{1}$, Han Shen ${ }^{2}$, Yuanyuan Zhang $^{3}$ and Jianhua Chen ${ }^{4}$ \\ ${ }^{1,2,3,4}$ School of Mathematic and Statistics, Wuhan University, Wuhan, Hubei, China \\ ${ }^{1}$ shiyj_ecc@163.com, ${ }^{2}$ Hjmshenhan_ecc@163.com, ${ }^{3}$ circle0519@hotmail.com
}

\begin{abstract}
To keep the pace with the development of internet technology, remote user authentication techniques become more and more important to protect user's privacy. Recently, Kumari, et al., presented an improved remote user authentication scheme with key agreement based on dynamic-identity using smart card. This scheme allows legal users to change the password at his will without the need to connect the server. They claimed that their scheme could resist smart card stolen or loss attack, user impersonation and server masquerading attack, and provide user anonymity and untraceability and so on. However, our research indicates that their scheme is completely unsafe. Furthermore, the scheme can't provide the proper mutual authentication. In this manuscript, we will propose a new scheme, which can withstand those attacks mentioned above and provide the perfect user anonymity and forward secrecy. Security analysis makes it clear that the improved scheme apparently is more secure and practical.
\end{abstract}

Keywords: key agreement, dynamic-identity, user anonymity and untraceability, smart card

\section{Introduction}

With the rapid development of network technology, remote user authentication has become an indispensable part of life to access to the worthy resources, which are provided by the host across the network by the remote systems. And before having the access to the services, people should be verified to be a legal user through the authentication of the remote system. Otherwise, an attacker could pretend to be a legal user to log in the system and get the resources. That is, only the legal user through the authentication of the remote system could have the access to the service, or an attacker could easily log in the system. As a result, researchers pay more attention on the secured issues and users' authentication.

The authentication techniques are essential to guarantee the users' legality. In 1981, Lamport [1] had come-up with a remote authentication based on password Table to authenticate the right of logging in the system. Later, many improved schemes are proposed on the security and efficiency [2-9]. However, those schemes need a Table of passwords or some values related to password stored by the server to complete the authentication. Once an adversary got the Table, the system would be partly or completely broken and users would suffer a lot.

To avoid the verified Table $[8,10,11]$, researchers proposed some schemes based on static ID of users' identities which could widely leak the information of the legal users. But adversary may obtain the ID and other massages to disguise as a user to log into the remote systems. And many static ID-based remote user authentications can't provide users with the convenience of password change or verify the validity of the login requests without any verify Table. To overcome these risks and improve the efficiency and security, many applications and researches [12-21] based on dynamic ID have been done. And owing to the features of portability, low cost, cryptographic and computational 
capacity, the smart cards are used in the scheme of authentication on a large scale.

In 2004, an authentication scheme based on a dynamic ID was proposed by Das, et al., and they claimed that their scheme was secure to resist ID-theft, replay attacks and insider attack. However, Wang, et al., [11] pointed out that the Das, et al., scheme didn't achieve mutual authentication and dependence of password, and could not resist impersonate server attack. Therefore, they advanced a new scheme to strengthen the password authentication and provide mutual authentication in 2009. But many researches [22-25] made it clear that the improved scheme was vulnerable .In 2009, Chang, et al., [22, 23] demonstrated that the scheme proposed by Wang wasn't efficient and secure because the attacker can be verified by the server and select an identity at his will. Then they provided a new scheme based on a smart card to complete the mutual authentication without any verified Table and they claimed that the scheme could provide untraceability and convenience of password update.

In 2013, Kumari, et al., [26] proposed an improved user authentication scheme with key agreement, then they pointed out that the scheme of Chang, et al., could not resist off-line password guessing attack, user impersonation attack, server masquerading attack, insider attack and so on. They showed that once smart card of any user was lost, the password of every legal user in the Chang, et al., scheme system will be at stake. With the information of any smart card, the adversary could pretend to be any user in this system and get the service of the server successfully. What's more, there were several loopholes in password change phase and no verification mechanism existed in smart card. Besides, the scheme didn't provide any session key agreement. Then Kumari, et al., presented an improved scheme and they claimed that the scheme could resist the smart card loss attack, impersonation attack, sever masquerading attack and provide forward secrecy, user anonymity and untraceability and so on.

In this article, however, we illustrate that Kumari, et al., scheme is still vulnerable to the smart card stolen or loss attack, forge attack and even fail to provide the forward secrecy and so on. The rest organization of the article is arranged as follows: Section 2 reviews Kumari et al., scheme. The cryptanalysis of Kumari, et al., scheme is shown in Section 3. Section 4 gives the details of our new proposed scheme. Section 5 makes the security analysis of the proposed scheme. Section 6 compares the performance and efficient of new scheme. At last, the conclusion of this paper will be given in Section7.

\section{Review of Kumari, et al., Scheme}

In this part, we review Kumari, et al., scheme, which consists of four phases: the registration phase, the login phase, authentication phase and password change phase. The detail steps will be shown as follows. The notations used in the whole literature are indicated in Table 1.

Table 1. Notations

\begin{tabular}{clll}
\hline Notations & & & \\
\hline$U_{i} / U_{e}$ & A system user/an attacker $E$ & $\|$ & A concatenation operation \\
$S_{i}$ & The remote server & $S C$ & Smart card \\
$I D_{i}, P w_{i}, P w_{\text {new }}$ & $U_{i}$ s identity, password, new password & $r_{i}$ & Unique random number assigned to $U_{i}$ by $S_{i}$ \\
$C I D_{i}$ & $U_{i}$ 's dynamic identity & $r$ & A random number selected by the smart card \\
$T_{1}, T_{2}, T_{3}$ & Timestamps & $\oplus$ & An XOR operation \\
$h()$. & A secure one-way hash function & $x(\mathrm{y})$ & A secret key(number) of server \\
\hline
\end{tabular}

\subsection{Registration Phase}

In this phase, we can know that if a user $U_{i}$ would like to become a legal user of this system $S_{i}, U_{i}$ should carry out the following steps. 
1) The user $U_{i}$ selects identity $I D_{i}$ and password $P W_{i}$ and chooses a random number $n$.Then $U_{i}$ computes $R_{i}=h\left(n \| P W_{i}\right)$ and sends $\left\{I D_{i}, R_{i}\right\}$ to $S_{i}$ via a secure channel.

2) After receiving $\left\{I D_{i}, R_{i}\right\}$, the server $S_{i}$ chooses a random number $r_{i}$ for each user $U_{i}$, which means that $r_{i}$ is different for every user. $x$ is the private key of $S_{i}$ and $y$ is a secrete number of $S_{i}$.

3) Then $S_{i}$ computes $T N_{i}=h\left(I D_{i} \| x\right) \oplus R_{i}, T Y_{i}=r_{i} \oplus h\left(I D_{i} \| x\right), T D_{i}=h\left(I D_{i}\left\|r_{i}\right\| R_{i}\right)$ and $T E_{i}=r_{i} \oplus h(y \| x)$.Then $S_{i}$ stores $\left\{T Y_{i}, T D_{i}, T E_{i}, h\right\}$ into smart card and issues $\left\{\right.$ smart card,$\left.T N_{i}\right\}$ to the user $U_{i}$ via a secure channel.

4) On receiving $\left\{\right.$ smart card, $\left.T N_{i}\right\}$ from the server $S_{i}$, the user $U_{i}$ computes $A_{i}=\left(I D_{i} \| P W_{i}\right) \oplus n, T M_{i}=T N_{i} \oplus n$ and stores $\left\{A_{i}, T M_{i}\right\}$ into smart card.

The registration phase of Kumari et al., scheme is showed in Figure 1.

Registration phase

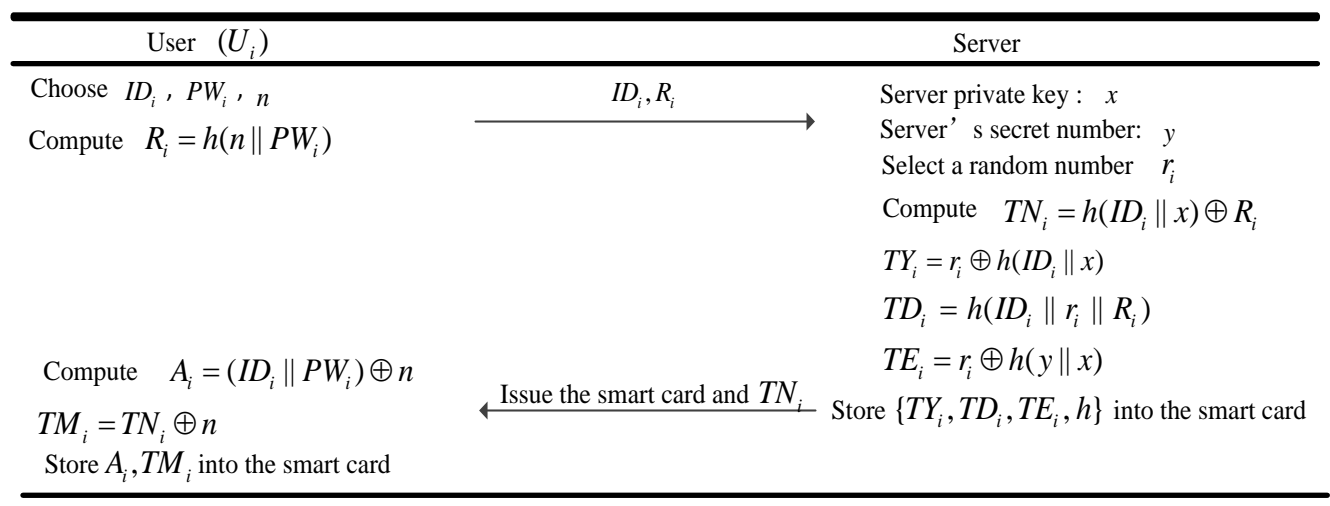

Figure 1. The Registration Phase of Kumari, et al., Scheme

\subsection{Login phase}

When user $U_{i}$ wants to get the service from $S_{i}$, he should perform the following steps.

1) The user $U_{i}$ inputs identity $I D_{i}$ and password $P W_{i}$, then the smart card SC computes $n=A_{i} \oplus\left(I D_{i} \| P W_{i}\right), R_{i}=h\left(n \| P W_{i}\right), h\left(I D_{i} \| x\right)=T M_{i} \oplus R_{i} \oplus n, r_{i}=T Y_{i} \oplus h\left(I D_{i} \| x\right)$ and checks whether the equation $T D_{i} ?=h\left(I D_{i}\left\|r_{i}\right\| R_{i}\right)$ holds or not.

2) If not, $\mathrm{SC}$ will drop the session. If it repeats thrice then $\mathrm{SC}$ gets blocked and $U_{i}$ is required to enter PUK to SC.

3) If holds, SC will compute $h(y \| x)=r_{i} \oplus T E_{i}, T N_{i}=T M_{i} \oplus n$ and gets the current time $T_{1}$.

4) $\mathrm{SC}$ computes $C I D_{i}=I D_{i} \oplus h\left(N_{i}\left\|r_{i}\right\| T_{1}\right), T N_{i}^{\prime}=T N_{i} \oplus h\left(r_{i} \| T_{1}\right), T B_{i}=T N_{i} \oplus R_{i}$, $T C_{i}=h\left(T N_{i}\left\|r_{i}\right\| T B_{i} \| T_{1}\right), T F_{i}=r_{i} \oplus\left(h(y \| x) \| T_{1}\right)$ and sends $\left\{C I D_{i}, T N_{i}^{\prime}, T C_{i}, T F_{i}, T_{1}\right\}$ to $S_{i}$ via a public channel.

\subsection{Authentication Phase}

When $S_{i}$ gets the message $\left\{C I D_{i}, T N_{i}^{\prime}, T C_{i}, T F_{i}, T_{1}\right\}$ from $U_{i}, S_{i}$ will play the detail steps as follows to finish the authentication phase and key agreement phase.

1) $S_{i}$ gets the current time $T_{2}$ and checks whether $T_{1}$ is valid or not, which means 
$T_{2}-T_{1} \leq \Delta T$.If $T_{1}$ isn't valid, $S_{i}$ rejects all login requests and drops this phase.

2) If $T_{1}$ is valid, $S_{i}$ continues to compute $r_{i}=T F_{i} \oplus\left(h(y \| x) \| T_{1}\right), T N_{i}=T N_{i}^{\prime} \oplus h\left(r_{i} \| T_{1}\right)$, $I D_{i}=C I \boxplus\left(h T_{i}\left|N_{i}\right|_{1} \mid r, T B_{i}^{*}=h\left(I D_{i} \| x\right)\right.$ and checks whether the equation $T C_{i} ?=h\left(T N_{i}\left\|r_{i}\right\| T B_{i}^{*} \| T_{1}\right)$ holds or not.

3) If holds, $S_{i}$ gets the current time $T_{3}$ and computes $A=h\left(T B_{i}^{*}\left\|r_{i}\right\| T_{3}\right)$.Then he sends $\left\{A, T_{3}\right\}$ to the user $U_{i}$.

4) After obtaining the $\left\{A, T_{3}\right\}$ from $S_{i}$, SC checks the validity of $T_{3}$.If $T_{3}$ is valid, the smart card will check the equation $A$ ? $=h\left(T B_{i}\left\|r_{i}\right\| T_{3}\right)$.If holds, the user and server complete the mutual authentication. Otherwise, the session will be terminated by $S_{i}$.

5) Finally, the user $U_{i}$ and the server $S_{i}$ negotiate the session key. $U_{i}$ computes the value of session key $S K=h\left(T B_{i}\left\|r_{i}\right\| T_{1}\left\|T_{3}\right\| h(y \| x)\right)$ and the server $S_{i}$ computes the value of session key $S K^{*}=h\left(T B_{i}^{*}\left\|r_{i}\right\| T_{1}\left\|T_{3}\right\| h(y \| x)\right)$ respectively. 2.

The login and authentication phases of Kumari et al., scheme are summarized in Figure

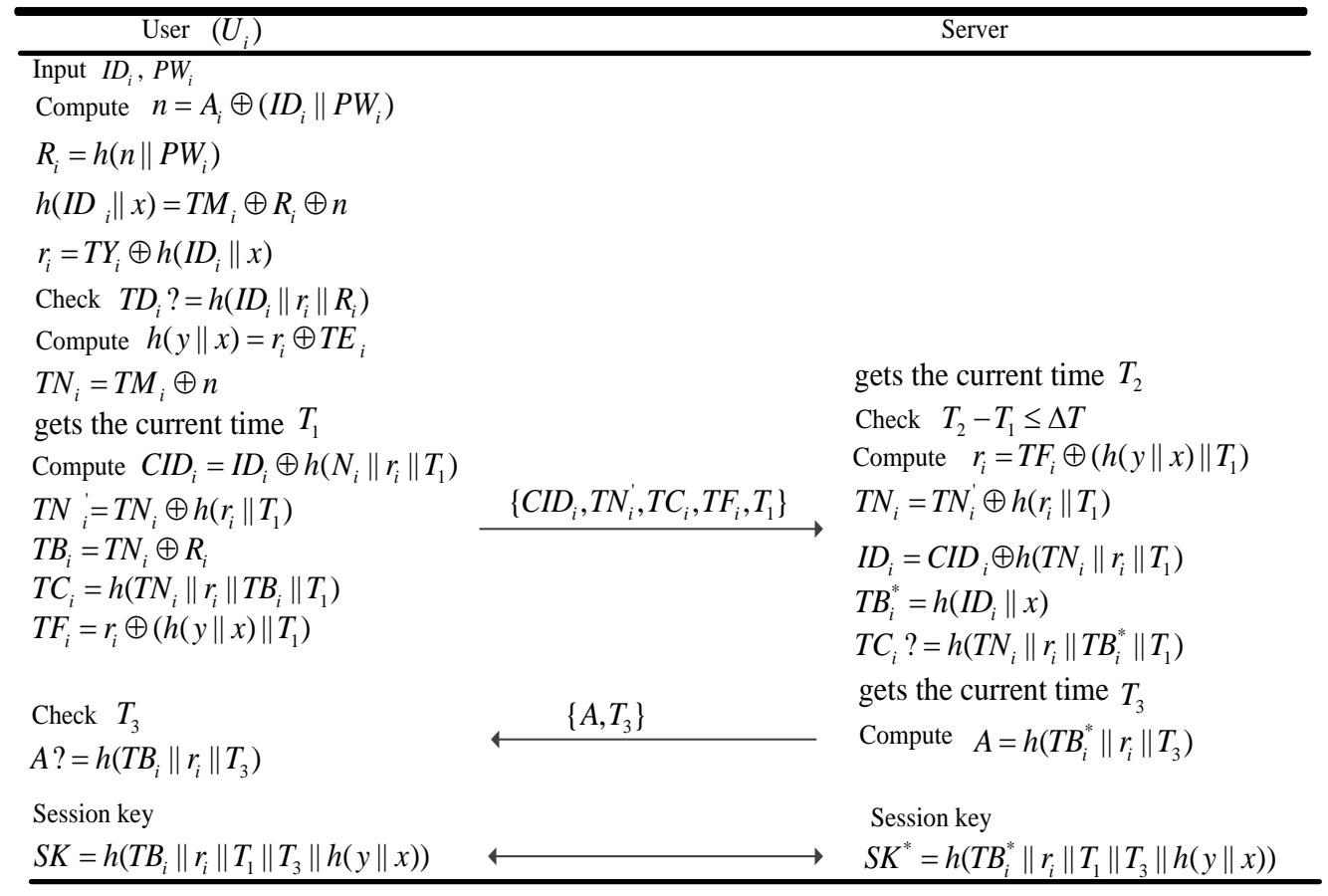

Figure 2. The Login and Authentication Phases of Kumari, et al., Scheme

\subsection{Password Change Phase}

If the user $U_{i}$ wants to change his password in the system, he must take such steps as follows.

1) $U_{i}$ inserts his smart card into the device and inputs his identity $I D_{i}$ and password $P W_{i}$ into the smart card.

2) Then SC computes $n=A_{i} \oplus\left(I D_{i} \| P W_{i}\right), R_{i}=h\left(n \| P W_{i}\right), \quad h\left(I D_{i} \| x\right)=T M_{i} \oplus R_{i} \oplus n$, $r_{i}=T Y_{i} \oplus h\left(I D_{i} \| x\right)$ and checks whether the equation $T D_{i} ?=h\left(I D_{i}\left\|r_{i}\right\| R_{i}\right)$ holds or not.

3) If holds, $\mathrm{SC}$ reminds the user of entering the new password $P W_{i}^{\text {new }}$ and computes the 


$$
\begin{aligned}
& \text { values } \quad \text { of } \quad R_{i}^{\text {new }}=h\left(n \| P W_{i}^{\text {new }}\right) \quad, \quad \mathrm{A}_{i}^{\text {new }}=h\left(I D_{i} \| P W_{i}^{\text {new }}\right) \oplus n, \\
& M_{i}^{\text {new }}=M_{i} \oplus R_{i} \oplus R_{i}^{\text {new }} \text { and } T D_{i}^{\text {new }}=h\left(I D_{i}\left\|r_{i}\right\| R_{i}^{\text {new }}\right) .
\end{aligned}
$$

4) Finally, $\mathrm{SC}$ replaces the old $\left\{A_{i}, T D_{i}, T M_{i}\right\}$ with $\left\{A_{i}^{\text {new }}, T D_{i}^{\text {new }}, T M_{i}^{\text {new }}\right\}$ respectively.

\section{Cryptanalysis of Kumari, et al., Scheme}

Kumari, et al., claimed that their proposed scheme can resist smart card loss attack, user impersonation, the server masquerading attack and so on. Besides, they could also provide user anonymity and untraceability. However, we find that their system actually fails to resist attacks mentioned above and can't provide user anonymity and untraceability. The detail steps of those attacks will be shown as follows.

\subsection{Smart Card Stolen or Loss Attack}

1) The attacker $E$ obtains $\left\{A_{e}, T M_{e}, T Y_{e}, T D_{e}, T E_{e}\right\}$ from his own smart card [27, 28] and

computes $\quad n_{e}=A_{e} \oplus\left(I D_{e} \| P w_{e}\right), \quad R_{e}=h\left(n_{e} \| P w_{e}\right), \quad h\left(I D_{e} \| x\right)=T M_{e} \oplus R_{e} \oplus n_{e} \quad$, $r_{e}=h\left(I D_{e} \| x\right) \oplus T Y_{e}, h(y \| x)=r_{e} \oplus T E_{e}$, so attacker $E$ can obtain the system value of $h(y \| x)$.

2) When the legal user $U_{i}$ logins the system and sends login request message $\left\{C I D_{i}, T N_{i}^{\prime}, T C_{i}, T F_{i}, T_{1}\right\}$.Then attacker $E$ can intercept the login request massage $\left\{C I D_{i}, T N_{i}^{\prime}, T C_{i}, T F_{i}, T_{1} \quad\right.$ and computes $r_{i}=T F_{i} \oplus\left(h(y \| x) \| T_{1}\right)$, $T N_{i}=T N_{i}^{\prime} \oplus h\left(r_{i} \| T_{1}\right), \quad I D_{i}=C I D_{i} \oplus h\left(T N_{i}\left\|r_{i}\right\| T_{1}\right)$ and $T E_{i}=r_{i} \oplus h(y \| x)$, which $h(y \| x)$ is computed from step (1).

3) Then we can know $\mathrm{H}:\left\{I D_{i}, r_{i}, T N_{i}, T E_{i}, h(y \| x)\right\}$ of every legal user $U_{i}$ from those computations. No matter what password is, $\left\{I D_{i}, r_{i}, T E_{i}, h(y \| x)\right\}$ are unchanged for each $U_{i}$ and $T N_{i}$ is fixed before $U_{i}$ 's passwords changed.

4) When $U_{i}$ 's smart card was stolen or lost, the attacker $E$ obtains the information $\left\{A_{i}, T M_{i}, T Y_{i}, T D_{i}, T E_{i}\right\}$ and the attackers searches $T E_{i}$ in $\mathrm{H}:\left\{I D_{i}, r_{i}, T N_{i}, T E_{i}, h(y \| x)\right\}$. Then the attackers can get $\left\{I D_{i}, r_{i}, T N_{i}\right\}$ of users.

5) The attackers computes $n_{i}=T M_{i} \oplus T N_{i}, \quad\left(I D_{i} \| P w_{i}\right)=A_{i} \oplus n_{i}$ and the attackers obtains $I D_{i}$ from step(3), then attacker can get $P w_{i}$ from $\left(I D_{i} \| P w_{i}\right)=A_{i} \oplus n_{i}$.

\subsection{User Impersonation}

If user $U_{i}$ ' smart card was stolen or lost, from section 3.1, the attacker $E$ can obtain $P w_{i}, \quad n_{i}, T M_{i}, R_{i}=h\left(n_{i} \| P W_{i}\right)$ and then computes $h\left(I D{ }_{i} \| x\right)=T M_{i} \oplus R_{i} \oplus n_{i}$.As we know, for each user $U_{i}$, the value of $h\left(I D{ }_{i} \| x\right)$ is unchanged, so after user $U_{i}$ get a new smart card with the same $I D_{i}$, the attacker can also obtain $\left\{I D_{i}, r_{i}, T E_{i}, h(y \| x), T N_{i}\right\}$ by intercepting previous message $\left\{C I D_{i}, T N_{i}^{\prime}, T C_{i}, T F_{i}, T_{1}\right\}$ and pretends to be user $U_{i}$ to login the system without the new smart card, the detail steps are shown as follows.

1) The attacker computes $C I D_{i}^{*}=I D_{i} \oplus h\left(T N_{i}\left\|r_{i}\right\| T_{1}\right), \quad T N_{i}^{*}=T N_{i} \oplus h\left(r_{i} \| T_{1}\right)$, $T B_{i}=h\left(I D_{i} \| x\right), T C_{i}^{*}=h\left(T N_{i}\left\|r_{i}\right\| T B_{i} \| T_{1}\right)$ and $T F_{i}^{*}=r_{i} \oplus\left(h(y \| x) \| T_{1}\right)$, which $h\left(I D_{i} \| x\right)$ is already known. Then attacker sends $\left\{C I D_{i}^{*}, T N_{i}^{*}, T C_{i}^{*}, T F_{i}^{*}, T_{1}\right\}$ to $S_{i}$. 
2) When $S_{i}$ receives the message $\left\{C I D_{i}^{*}, T N_{i}^{*}, T C_{i}^{*}, T F_{i}^{*}, T_{1}\right\}$ from the attacker, $S_{i}$ gets the current time $T_{2}$ and checks whether $T_{1}$ is valid, which means $T_{2}-T_{1} \leq \Delta T$.If $T_{1}$ isn't valid, $S_{i}$ rejects all login requests and drops this phase.

3) If $T_{1}$ is valid, $S_{i}$ computes the values of $\quad r_{i}=T F_{i}^{*} \oplus\left(h(y \| x) \| T_{1}\right) \quad, \quad T N_{i}=T N_{i}^{*} \oplus h\left(r_{i} \| T_{1}\right)$ $I D_{i}=C I D_{i}{ }^{*} \oplus h\left(T N_{i}\left\|r_{i}\right\| T_{1}\right), T B_{i}^{*}=h\left(I D_{i} \| x\right)$ and checks whether the equation $T C_{i}^{*} ?=h\left(T N_{i}\left\|r_{i}\right\| T B_{i}^{*} \| T_{1}\right)$ holds or not.

4) As we already know, $T C_{i}^{*}=h\left(T N_{i}\left\|r_{i}\right\| T B_{i} \| T_{1}\right)$ and the equation $T B_{i}=h\left(I D_{i} \| x\right)=T B_{i}^{*}$ holds, and then we can get $T C_{i}^{*}=h\left(T N_{i}\left\|r_{i}\right\| T B_{i}^{*} \| T_{1}\right) . S_{i}$ gets the current time $T_{3}$ and computes $A=h\left(T B_{i}^{*}\left\|r_{i}\right\| T_{3}\right)$, and then $S_{i}$ sends $\left\{A, T_{3}\right\}$ to SC. And the attacker $E$ has successfully login the system as a legal user and the session key is $S K^{* *}=h\left(T B_{i}\left\|r_{i}\right\| T_{1}\left\|T_{3}\right\| h(y \| x)\right)$.

\subsection{Server Masquerading Attack}

The attacker gets $\left\{h\left(I D_{i} \| x\right), h(y \| x)\right\}$ by using the method mentioned above in section 3.1 and 3.2.

1) When the user $U_{i}$ sends the login request message $\left\{C I D_{i}, T N_{i}^{\prime}, T C_{i}, T F_{i}, T_{1}\right\}$ in the public channel, the attacker can eavesdrop the login message and computes the values $r_{i}=T F_{i} \oplus\left(h(y \| x) \| T_{1}\right) \quad, \quad T N_{i}=T N_{i}^{\prime} \oplus h\left(r_{i} \| T_{1}\right) \quad, \quad I D_{i}=C I D_{i} \oplus h\left(T N_{i}\left\|r_{i}\right\| T_{1}\right) \quad$ and $T B_{i}^{*}=h\left(I D_{i} \| x\right)$, which $h\left(I D_{i} \| x\right)$ is already known from section 3.2.

2) The attacker gets the current time $T_{3}$ and computes $A=h\left(T B_{i}^{*}\left\|r_{i}\right\| T_{3}\right)$.Then he sends $\left\{A, T_{3}\right\}$ to $\mathrm{SC}$.

3) When $U_{i}$ receives the message from the attacker, he checks $T_{3}$ and verify the equation $A ?=h\left(T B_{i}\left\|r_{i}\right\| T_{3}\right)$.As we know $T B_{i}=h\left(I D_{i} \| x\right)=T B_{i}^{*}$, then the equation will hold.

4) Finally, the user and the attacker complete the authentication, and the user computes the session key $S K=h\left(T B_{i}\left\|r_{i}\right\| T_{1}\left\|T_{3}\right\| h(y \| x)\right)$ and the server computes the session key $S K^{*}=h\left(T B_{i}^{*}\left\|r_{i}\right\| T_{1}\left\|T_{3}\right\| h(y \| x)\right)$ respectively.

\subsection{User's Identity is Traceable}

1) The attacker $E$ obtains $\left\{A_{e}, T M_{e}, T Y_{e}, T D_{e}, T E_{e}\right\}$ from his own smart card and then he computes $\quad n_{e}=A_{e} \oplus\left(I D_{e} \| P w_{e}\right), \quad R_{e}=h\left(n_{e} \| P w_{e}\right), \quad h\left(I D_{e} \| x\right)=T M_{e} \oplus R_{e} \oplus n_{e} \quad$, $r_{e}=h\left(I D_{e} \| x\right) \oplus T Y_{e}, h(y \| x)=r_{e} \oplus T E_{e}$, so attacker $E$ can easily get the system value of $h(y \| x)$.

2) When the legal user $U_{i}$ logins the system and sends login request message $\left\{C I D_{i}, T N_{i}^{\prime}, T C_{i}, T F_{i}, T_{1}\right\}$ to the server, then attacker $E$ can intercept the login request massage $\left\{C I D_{i}, T N_{i}^{\prime}, T C_{i}, T F_{i}, T_{1} \quad\right\}$ and computes $r_{i}=T F_{i} \oplus\left(h(y \| x) \| T_{1}\right)$, $T N_{i}=T N_{i}^{\prime} \oplus h\left(r_{i} \| T_{1}\right), I D_{i}=C I D_{i} \oplus h\left(T N_{i}\left\|r_{i}\right\| T_{1}\right)$. Then he can continue to compute $T E_{i}=r_{i} \oplus h(y \| x)$, which $h(y \| x)$ is computed from step (1).

3) Then we know $\mathrm{H}:\left\{I D_{i}, r_{i}, T N_{i}, T E_{i}, h(y \| x)\right\}$ of each user $U_{i}$. No matter what 
password is, $\left\{I D_{i}, r_{i}, T E_{i}, h(y \| x)\right\}$ are unchanged for each $U_{i}$. So the attacker can get every user $U_{i}$ 's identity $I D_{i}$ in the system.

\subsection{Lack of the Forward Secrecy}

From section 3.1 and 3.2, the attacker $E$ can obtain the values of $\left\{I D_{i}, r_{i}, T E_{i}, h(y \| x), T N_{i}\right\}$ and gets $\left\{h\left(I D_{i} \| x\right), h(y \| x)\right\}$.If attacker $E$ had intercepted the message $\left\{C I D_{i}, T N_{i}^{\prime}, T C_{i}, T F_{i}, T_{1}\right\}$ and $\left\{A, T_{3}\right\}$ every time, he could get previous session key by computing the value of $S K^{* *}=h\left(h\left(I D_{i} \| x\right)\left\|r_{i}\right\| T_{1}\left\|T_{3}\right\| h(y \| x)\right)$.

\section{The Proposed Scheme}

In this part, we proposed a new scheme to resist those attacks, which consists of four phases: the registration phase, the login phase, authentication phase and password change phase. The detail steps will be shown as follows.

\subsection{Registration Phase}

In this phase, we can know that if a user $U_{i}$ would like to become a legal user of this system $S_{i}, U_{i}$ should carry out the following steps.

Registration phase

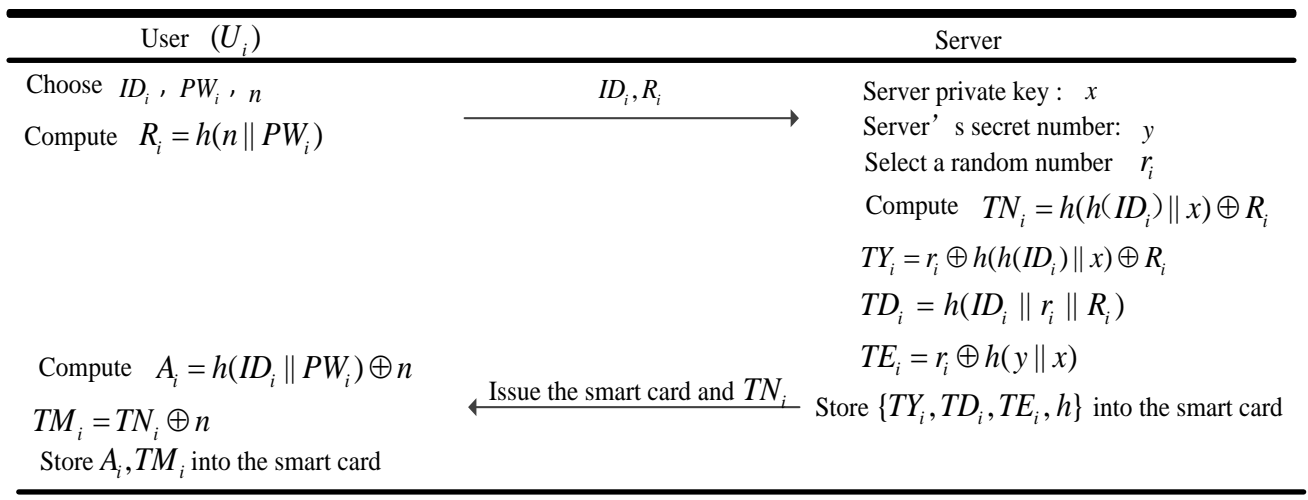

Figure 3. The Proposed Scheme of Registration Phase

1) $U_{i}$ chooses identity $I D_{i}$ and password $P W_{i}$, and then selects a random number $n$.he computes $R_{i}=h\left(n \| P W_{i}\right)$ and sends $\left\{I D_{i}, R_{i}\right\}$ to $S_{i}$ via a secure channel.

2) After receiving $\left\{I D_{i}, R_{i}\right\}, S_{i}$ chooses a random number $r_{i}$ for each user $U_{i}$, which means that $r_{i}$ is different for every user. $x$ is the private key of $S_{i}$ and $y$ is a secret number of $S_{i}$.

3) Then $S_{i}$ computes the values of $T N_{i}=h\left(h\left(I D_{i}\right) \| x\right) \oplus R_{i}, T Y_{i}=r_{i} \oplus h\left(h\left(I D_{i}\right) \| x\right) \oplus R_{i}$, $T D_{i}=h\left(I D_{i}\left\|r_{i}\right\| R_{i}\right)$ and $T E_{i}=r_{i} \oplus h(y \| x)$.Then he stores $\left\{T Y_{i}, T D_{i}, T E_{i}, h\right\}$ into smart card and issues $\left\{\right.$ smart card,$\left.T N_{i}\right\}$ to the user $U_{i}$ via a secure channel.

4) On receiving $\left\{\right.$ smart card, $\left.T N_{i}\right\}$ from $S_{i}$, the user $U_{i}$ computes $A_{i}=h\left(I D_{i} \| P W_{i}\right) \oplus n$, $T M_{i}=T N_{i} \oplus n$ and stores $\left\{A_{i}, T M_{i}\right\}$ into smart card.

\subsection{Login Phase}


When user $U_{i}$ wants to get the service from $S_{i}$, he should perform the following steps.

1) $U_{i}$ inputs identity $I D_{i}$ and password $P W_{i}$.Then the smart card SC computes the values of $\quad n=A_{i} \oplus h\left(I D_{i} \| P W_{i}\right), \quad R_{i}=h\left(n \| P W_{i}\right) \quad$ and $\quad h\left(h\left(I D_{i}\right) \| x\right)=T M_{i} \oplus R_{i} \oplus n$, $r_{i}=T Y_{i} \oplus h\left(h\left(I D_{i}\right) \| x\right) \oplus R_{i}$.

2) $\mathrm{SC}$ checks whether the equation $T D_{i} ?=h\left(I D_{i}\left\|r_{i}\right\| R_{i}\right)$ holds or not. If not, SC drops the session. If holds, SC computes $h(y \| x)=r_{i} \oplus T E_{i}, T N_{i}=T M_{i} \oplus n$ and gets the current time $T_{1}$.

3) $\mathrm{SC}$

computes $C I D_{i}=h\left(I D_{i}\right) \oplus h\left(N_{i}\left\|r_{i}\right\| T_{1}\right), T N_{i}^{\prime}=T N_{i} \oplus h\left(r_{i} \| T_{1}\right), T B_{i}=T N_{i} \oplus R_{i}$ and chooses a random number $r$.Then SC can compute $Q_{i}=h\left(h\left(I D_{i}\right) \| r\right), D S_{i}=T B_{i} \oplus Q_{i}$, $T C_{i}=h\left(T N_{i}\left\|r_{i}\right\| Q_{i} \| T_{1}\right) \quad, \quad T F_{i}=r_{i} \oplus\left(h(y \| x) \| T_{1}\right) \quad$ and sends $\left\{C I D_{i}, T N_{i}^{\prime}, T C_{i}, T F_{i}, T_{1}, D S_{i}\right\}$ to $S_{i}$ via a public channel.

Login phase and authentication phase

\begin{tabular}{|c|c|c|}
\hline User $\left(U_{i}\right)$ & & Server \\
\hline \multicolumn{3}{|l|}{$\begin{array}{l}\text { Input } I D_{i}, P W_{i} \\
\text { Compute } n=A_{i} \oplus h\left(I D_{i} \| P W_{i}\right)\end{array}$} \\
\hline \multicolumn{3}{|l|}{$R_{i}=h\left(n \| P W_{i}\right)$} \\
\hline \multicolumn{3}{|l|}{$T B_{i}=h\left(h\left(I D_{i}\right) \| x\right)=T M_{i} \oplus R_{i} \oplus n$} \\
\hline \multicolumn{3}{|l|}{$r_{i}=T Y_{i} \oplus h\left(h\left(I D_{i}\right) \| x\right) \oplus R_{i}$} \\
\hline \multicolumn{3}{|l|}{ Check $T D_{i} ?=h\left(I D_{i}\left\|r_{i}\right\| R_{i}\right)$} \\
\hline$T N_{i}=T M_{i} \oplus n$ & & gets the current time $T_{2}$ \\
\hline \multicolumn{2}{|l|}{$\begin{array}{l}\text { gets the current time } T_{1} \\
\text { Compute } C I D_{i}=h\left(I D_{i}\right) \oplus h\left(N_{i}\left\|r_{i}\right\| T_{1}\right)\end{array}$} & Check $T_{2}-T_{1} \leq \Delta T$ \\
\hline \multicolumn{3}{|l|}{ 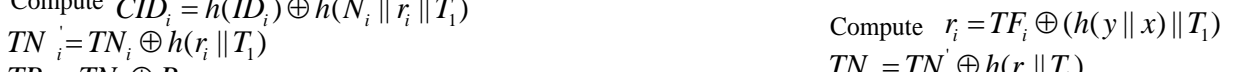 } \\
\hline $\begin{array}{l}T B_{i}=T N_{i} \oplus R_{i} \\
\text { Generate a random nuber } \quad r\end{array}$ & & $\begin{array}{l}T N_{i}=T N_{i} \oplus h\left(r_{i} \| T_{1}\right) \\
h\left(I D_{i}\right)=C I D_{i} \oplus h\left(T N_{i}\left\|r_{i}\right\| T_{1}\right)\end{array}$ \\
\hline Compute $Q_{i}=h\left(h\left(I D_{i}\right) \| r\right)$ & $\left\{C I D_{i}, T N_{i}, T C_{i}, T F_{i}, I_{1}, D S_{i}\right\}$ & $T B_{i}^{*}=h\left(h\left(I D_{i}\right) \| x\right)$ \\
\hline $\begin{aligned} D S_{i} & =T B_{i} \oplus Q_{i} \\
T C_{i} & =h\left(T N_{i}\left\|r_{i}\right\| Q_{i} \| T_{1}\right)\end{aligned}$ & & $Q_{i}^{*}=T B_{i}^{*} \oplus D S_{i}$ \\
\hline$T F_{i}=r_{i} \oplus\left(h(y \| x) \| T_{1}\right)$ & & $\begin{array}{l}T C_{i} ?=h\left(T N_{i}\left\|r_{i}\right\| Q_{i}^{*} \| T_{1}\right) \\
\text { gets the current time } T_{3}\end{array}$ \\
\hline $\begin{array}{l}\text { Check } T_{3} \\
A ?=h\left(T B_{i}\left\|r_{i}\right\| T_{3}\right)\end{array}$ & $\left\{A, T_{3}\right\}$ & Compute $A=h\left(T B_{i}^{*}\left\|r_{i}\right\| T_{3}\right)$ \\
\hline Session key & $\longleftrightarrow$ & Session key \\
\hline$S K=h\left(T B_{i}\left\|r_{i}\right\| T_{1}\left\|T_{3}\right\| h(y \| x) \| Q_{i}\right)$ & & $K^{*}=h\left(T B_{i}^{*}\left\|r_{i}\right\| T_{1}\left\|T_{3}\right\| h(y \| x) \| Q_{i}^{*}\right)$ \\
\hline
\end{tabular}

Figure 4. The Proposed Scheme of Login and Authentication Phase

\subsection{Authentication Phase}

When $S_{i}$ gets the message $\left\{C I D_{i}, T N_{i}^{\prime}, T C_{i}, T F_{i}, T_{1}, D S_{i}\right\}$ from $U_{i}, S_{i}$ will play the detail steps as follows to finish the authentication phase and key agreement phase.

1) $S_{i}$ gets the current time $T_{2}$ and checks whether $T_{1}$ is valid, which means $T_{2}-T_{1} \leq \Delta T$.If $T_{1}$ isn't valid, $S_{i}$ rejects all login requests and drops this phase.

2) If $T_{1}$ is valid, $S_{i}$ continues to compute $r_{i}=T F_{i} \oplus\left(h(y \| x) \| T_{1}\right), T N_{i}=T N_{i}^{\prime} \oplus h\left(r_{i} \| T_{1}\right)$, $h\left(I D_{i}\right)=C I D_{i} \oplus h\left(T N_{i}\left\|r_{i}\right\| T_{1}\right), T B_{i}^{*}=h\left(h\left(I D_{i}\right) \| x\right), Q_{i}^{*}=T B_{i}^{*} \oplus D S_{i}$, and checks 
whether the equation $T C_{i} ?=h\left(T N_{i}\left\|r_{i}\right\| Q_{i}^{*} \| T_{1}\right)$ holds or not.

3) If holds, $S_{i}$ gets the current time $T_{3}$ and computes $A=h\left(T B_{i}^{*}\left\|r_{i}\right\| T_{3}\right)$.Finally he sends $\left\{A, T_{3}\right\}$ to the user $U_{i}$.

4) After obtaining the $\left\{A, T_{3}\right\}$ from $S_{i}$, SC checks the validity of $T_{3}$. If $T_{3}$ is valid, checks weather the equation $A ?=h\left(T B_{i}\left\|r_{i}\right\| T_{3}\right)$ holds or not further. If holds, the user and server complete the mutual authentication. Otherwise, the session will be terminated by $S_{i}$.

5) Finally, the user $U_{i}$ and the server $S_{i}$ negotiate the session key. The user $U_{i}$ computes the session key $S K=h\left(T B_{i}\left\|r_{i}\right\| T_{1}\left\|T_{3}\right\| h(y \| x) \| Q_{i}\right)$ and the server computes the session key $S K^{*}=h\left(T B_{i}^{*}\left\|r_{i}\right\| T_{1}\left\|T_{3}\right\| h(y \| x) \| Q_{i}^{*}\right)$ respectively.

In the end, users can use the session key to encrypt or decrypt their massages for secret communication safely through a secure channel. Besides, the login and authentication phases of our proposed scheme are summarized in Figure 4.

\subsection{Password Change Phase}

If the user $U_{i}$ wants to change his password in the system, he must take such steps as follows. And the proposed scheme of password change phase is summarized in Figure 5.

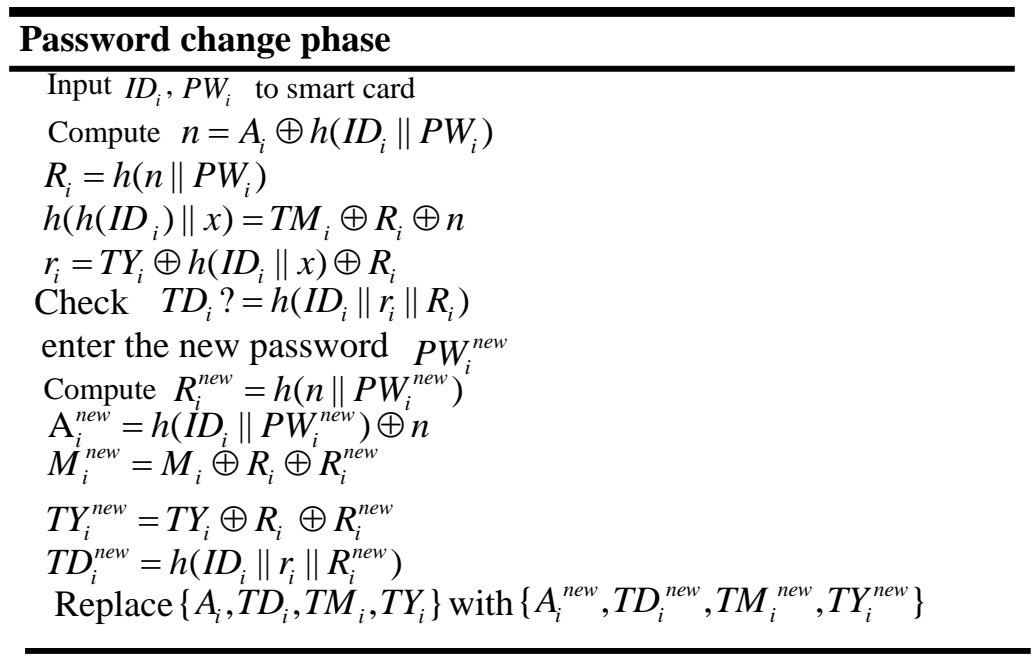

Figure 5. The Proposed Scheme of Password Change Phase

1) Inserts his smart card into the device, and then inputs his identity $I D_{i}$ and password $P W_{i}$ into the smart card. Then SC computes the values of $n=A_{i} \oplus h\left(I D_{i} \| P W_{i}\right)$, $R_{i}=h\left(n \| P W_{i}\right), h\left(h\left(I D_{i}\right) \| x\right)=T M_{i} \oplus R_{i} \oplus n, r_{i}=T Y_{i} \oplus h\left(I D_{i} \| x\right) \oplus R_{i}$ and checks whether the equation $T D_{i} ?=h\left(I D_{i}\left\|r_{i}\right\| R_{i}\right)$ holds or not.

2) If holds, SC reminds the user of entering the new password $P W_{i}^{\text {new }}$.Then $\mathrm{SC}$ computes

$R_{i}^{\text {new }}=h\left(n \| P W_{i}^{\text {new }}\right) \quad, \quad \mathrm{A}_{i}^{\text {new }}=h\left(I D_{i} \| P W_{i}^{\text {new }}\right) \oplus n \quad, \quad M_{i}^{\text {new }}=M_{i} \oplus R_{i} \oplus R_{i}^{\text {new }} \quad$, $T Y_{i}^{\text {new }}=T Y_{i} \oplus R_{i} \oplus R_{i}^{\text {new }} \quad, \quad T D_{i}^{\text {new }}=h\left(I D_{i}\left\|r_{i}\right\| R_{i}^{\text {new }}\right) \quad$ and $\quad$ replaces the old $\left\{A_{i}, T D_{i}, T M_{i}, T Y_{i}\right\}$ with $\left\{A_{i}^{\text {new }}, T D_{i}^{\text {new }}, T M_{i}^{\text {new }}, T Y_{i}^{\text {new }}\right\}$ respectively.

\section{Security Analysis of the Proposed Scheme}


In this section, we will show that our scheme can overcome the drawbacks analyzed above and further can resist some other attacks. The details are shown in the following.

\subsection{Users' Anonymity and Untraceability}

We will show that any attacker can trace the identity $I D_{i}$ of a user in the proposed scheme.

1) The attacker $E$ obtains $\left\{A_{e}, T M_{e}, T Y_{e}, T D_{e}, T E_{e}\right\}$ from his own smart card and then computes $n_{e}=A_{e} \oplus h\left(I D_{e} \| P w_{e}\right), \quad R_{e}=h\left(n_{e} \| P w_{e}\right), \quad h\left(h\left(I D_{e}\right) \| x\right)=T M_{e} \oplus R_{e} \oplus n_{e}$, $r_{e}=h\left(h\left(I D_{e}\right) \| x\right) \oplus T Y_{e} \oplus R_{e}, h(y \| x)=r_{e} \oplus T E_{e}$,so attacker $E$ can obtain the system value of $h(y \| x)$.

2) When the legal user $U_{i}$ logins the system and sends login request message $\left\{C I D_{i}, T N_{i}^{\prime}, T C_{i}, T F_{i}, T_{1}\right\}$, the attacker $E$ intercepts the massage $\left\{C I D_{i}, T N_{i}^{\prime}, T C_{i}, T F_{i}, T_{1}\right.$ \}and continues to compute the values of $r_{i}=T F_{i} \oplus\left(h(y \| x) \| T_{1}\right), T N_{i}=T N_{i}^{\prime} \oplus h\left(r_{i} \| T_{1}\right), \quad h\left(I D_{i}\right)=C I D_{i} \oplus h\left(T N_{i}\left\|r_{i}\right\| T_{1}\right)$. So the attacker just can get $h\left(I D_{i}\right)$ instead of $I D_{i}$ of a user in this system. Namely, this scheme can provide users' anonymity and untraceability.

\subsection{Off-line Password Guessing Attack}

Off-line password guessing attack means the attacker can use user's smart card to get the user's password off-line. In this scheme, the attacker stole a user's smart card. From the section 3.1, the attacker can get $\left\{n_{i}\right\}$ and computes $A_{i} \oplus n_{i}=h\left(I D_{i} \| P W_{i}\right)$. From the $h\left(I D_{i} \| P W_{i}\right)$, he can't guess the correct password without knowing $I D_{i}$.

\subsection{Smart Card Stolen or Loss Attack}

Suppose the attacker $E$ obtains the smart card of any user, we will show that $E$ can't get any useful information out of those extracted values. From section 5.1 and 5.2, we can know the values of $\left\{h\left(I D_{i}\right), T N_{i}, r_{i}, n_{i}\right\}$ from the attacks but not $\left\{I D_{i}, P W_{i}\right\}$, so the attackers can't compute $T B_{i}=h\left(h\left(I D_{i}\right) \| x\right)$ from the entities he has already known. Then he can't obtain the correct value of $D S_{i}=T B_{i} \oplus Q_{i}$ without correct value of $T B_{i}$. When the server receives the mock massage $\left\{C I D_{i}, T N_{i}^{\prime}, T C_{i}^{* * *}, T F_{i}, T_{1}, D S_{i}^{* * *}\right\}$ from the attacker, the server will reject the login request because the equation $T C_{i}^{* *} ?=T C_{i}^{*}$ won't hold with different $T B_{i}$. Therefore, the scheme can resist the smart card stolen or loss attack.

\subsection{Resist Server Masquerading and user Impersonation Attack}

If the attacker $E$ wants to impersonate a legal user of the system, the attacker $E$ needs to compute a correct login request $\left\{C I D_{i}, T N_{i}^{\prime}, T C_{i}, T F_{i}, T_{1}, D S_{i}\right\}$. However, based on the analysis of section 5.3, the attacker can't pass the authentication equation of $T C_{i}^{* *}=T C_{i}^{*}$ successfully without $T B_{i}^{*}$. So the scheme can resist the user impersonation attack. In order to masquerade server, the attacker should compute the valid value of $A=h\left(T B_{i}^{*}\left\|r_{i}\right\| T_{3}\right)$, but he can't get the private key $x$ or $T B_{i}$. Therefore, the scheme can resist server masquerading attack.

\subsection{Provides Forward Secrecy}

Forward secrecy is a very important part of ensuring the security of users' information. 
In the proposed scheme, the session key $\quad S K=h\left(h\left(I D_{i} \| x\right)\left\|r_{i}\right\| T_{1}\left\|T_{3}\right\| h(y \| x) \| h\left(h\left(I D_{i}\right) \| r\right)\right) \quad$ is $\quad$ based $\quad$ on $\left\{h\left(I D_{i} \| x\right), r_{i}, h(y \| x), h\left(h\left(I D_{i}\right) \| r\right)\right\}$, which means the adversary must know the value of $h\left(h\left(I D_{i}\right) \| r\right)$. Suppose the adversary can obtain $\left\{h\left(I D_{i} \| x\right), r_{i}, h(y \| x), h\left(I D_{i}\right)\right\}$, but he still won't get the number $r$, which is generated randomly in every round. Therefore, attacker can't compute the previous session keys properly.

\subsection{Resist Replay Attack}

The improved scheme uses the current timestamps to resist the relay attack. Namely, upon receiving the login request message $\left\{C I D_{i}, T N_{i}^{\prime}, T C_{i}, T F_{i}, T_{1}, D S_{i}\right\}$ from users, the server checks the validity of $T_{1}$ immediately. Similarly, the user receives the response message $\left\{A, T_{3}\right\}$ from the server and then verifies the legality of $T_{3}$ firstly. If the timestamps was out of deadline, the server or users will reject those request messages. So, the scheme can resist replay attack.

\subsection{Insider Attack}

In the improved scheme, users transmit a nonce $R_{i}$ instead of the plaintext password. To protect the password from insider attack, user $U_{i}$ uses a random number $n$ and submits the value of $R_{i}=h\left(n \| P W_{i}\right)$. But insiders don't know both of $\left\{n, P W_{i}\right\}$, this is, it is less likely to guess the two value in polynomial time. Therefore, the attacker doesn't have the chance to guess the probable password and test his guess right. Namely, this scheme can provide full resistance of insider attack.

\subsection{Provides Mutual Authentication}

In the proposed scheme, the server verifies the legal user by the method of checking the validity of equation $T C_{i} ?=h\left(T N_{i}\left\|r_{i}\right\| Q_{i}^{*} \| T_{1}\right)$.On the contrary, the user verifies the equation $A ?=h\left(T B_{i}\left\|r_{i}\right\| T_{3}\right)$ to authenticate the server. By the way, based on the analysis of section 5.4, no one can pretend to be a legal user or server to deceive others. So, the scheme can provide proper mutual authentication.

\subsection{Provides Session Key}

In the improved scheme, the user $U_{i}$ computes $S K=h\left(T B_{i}\left\|r_{i}\right\| T_{1}\left\|T_{3}\right\| h(y \| x) \| Q_{i}\right)$ and the server computes the session key $S K^{*}=h\left(T B_{i}^{*}\left\|r_{i}\right\| T_{1}\left\|T_{3}\right\| h(y \| x) \| Q_{i}^{*}\right)$ respectively at the end of every session, which is based on the fact that the equation $S K=h\left(h\left(I D_{i} \| x\right)\left\|r_{i}\right\| T_{1}\left\|T_{3}\right\| h(y \| x) \| h\left(h\left(I D_{i}\right) \| r\right)\right)$ holds. Then the user can transmit any information he wants to the server and the server can also provide any service he can to the legal users confidentially. Based on the rigorous analysis of section 5.5, the scheme can provide forward secrecy. That is, the scheme can provide reasonably and secure session key.

\section{Performance and Efficient Comparison}

In this section, we will compare the proposed scheme with Kumari, et al., Chang, et al., and Chou, et al., [29] on the aspects of performance and efficiency. The first Table will exhibit us that ours can resist all attacks mentioned in the Table 2.

The third Table will show the efficiency of those four schemes. In each scheme, there 
are four parts, which compose of the registration phase, the login phase, authentication phase and the total computational complexity. Notations used in the Table 1 will be described as follows:

$$
\begin{aligned}
& t_{\oplus}: \text { The complexity of one-way hash function operation } \\
& t_{h(.)}: \text { The complexity of XOR operation } \\
& t_{c e}: \text { The complexity of checking an equation }
\end{aligned}
$$

From the Table 2, we can know that our proposed scheme is obviously more efficient than other four schemes in the login and authentication phase on the foundation of safety. What's more, our scheme can change password without the need to connecting the server and taking no consideration of internet's condition. Besides, registration operation only needs to be carried out one time for every legal user, but the login and authentication phase is more likely to take place one more times in a short time. Thus, ours is more

\begin{tabular}{|c|c|c|c|c|}
\hline & Chou et al., & Chang et al., & Kumari et al., & Ours \\
\hline $\begin{array}{l}\text { registration } \\
\text { phase }\end{array}$ & $5 t_{\oplus}+8 t_{h()}$ & $t_{\oplus}+t_{h(\cdot)}$ & $5 t_{\oplus}+5 t_{h()}$ & $6 t_{\oplus}+8 t_{h()}$ \\
\hline login phase & $3 t_{\oplus}+6 t_{h()}$ & $3 t_{\oplus}+4 t_{h(.)}$ & $10 t_{\oplus}+8 t_{h(\cdot)}+t_{c \varepsilon}$ & $11 t_{\oplus}+12 t_{h(\cdot)}+t_{c s}$ \\
\hline $\begin{array}{l}\text { authentication } \\
\text { phase }\end{array}$ & $14 t_{\oplus}+20 t_{h(\cdot)}+3 t_{c s}$ & $6 t_{\oplus}+10 t_{h())}+2 t_{o s}$ & $3 t_{\oplus}+7 t_{h())}+2 t_{c \varepsilon}$ & $4 t_{\oplus}+8_{h(\cdot)}+2 t_{c \varepsilon}$ \\
\hline $\begin{array}{l}\text { total of login } \\
\text { and } \\
\text { authentication }\end{array}$ & $17 t_{\oplus}+26 t_{h(\cdot)}+3 t_{c \varepsilon}$ & $8 t_{\oplus}+14 t_{h(.)}+2 t_{\infty}$ & $13 t_{\oplus}+15 t_{h(.)}+3 t_{c e}$ & $15 t_{\oplus}+20 t_{h(.)}+3 t_{c \varepsilon}$ \\
\hline $\begin{array}{l}\text { Password } \\
\text { change }\end{array}$ & $6 t_{\oplus}+14 t_{h(\cdot)}+t_{c \varepsilon}$ & $8 t_{\oplus}+12 t_{h(\cdot)}+2 t_{c s}$ & $6 t_{\oplus}+6 t_{h()}+t_{c \varepsilon}$ & $10 t_{\oplus}+7 t_{h(.)}+t_{c s}$ \\
\hline $\begin{array}{l}\text { total } \\
\text { complexity }\end{array}$ & $28 t_{\oplus}+48 t_{h(\cdot)}+4 t_{c \varepsilon}$ & $18 t_{\oplus}+27 t_{h(.)}+4 t_{c \varepsilon}$ & $24 t_{\oplus}+26 t_{h(\cdot)}+4 t_{c \varepsilon}$ & $31 t_{\oplus}+35 t_{h(\cdot)}+4 t_{c \varepsilon}$ \\
\hline
\end{tabular}
convenient and practical than other three schemes.

Table 2. The Efficiency Comparison

In Table 3, we will display the security properties referring to security analysis among those four schemes.

Table 3. The Performance Comparison

\begin{tabular}{|c|c|c|c|c|}
\hline & Chou, et al., & Chang, et al., & Kumari, et al., & Ours \\
\hline Users' anonymity and untraceability. & No & No & No & Yes \\
\hline Off-line password guessing attack & No & No & Yes & Yes \\
\hline Insider attack & No & No & Yes & Yes \\
\hline Smart card stolen or loss attack & No & No & No & Yes \\
\hline server masquerading attack & Yes & No & No & Yes \\
\hline user impersonation attack & No & No & No & Yes \\
\hline Provides forward secrecy & Yes & N/A & No & Yes \\
\hline replay attack & Yes & Yes & Yes & Yes \\
\hline proper mutual authentication & No & No & No & Yes \\
\hline Provides session key & Yes & Yes & Yes & Yes \\
\hline Provides secure session key & No & N/A & No & Yes \\
\hline Total of No & 6 & $7+2(\mathrm{~N} / \mathrm{A})$ & 7 & 0 \\
\hline
\end{tabular}

From the Table 3, our proposed scheme is more secure than other three schemes, which are similar with each other among the login and authentication phases. And ours can resist 
all attacks mentioned above, but each of other three schemes has its own drawbacks.

\section{Conclusions}

In this study, we have reviewed the Kumari, et al., scheme with basic secure analysis, which described an improved remote user authentication scheme with key agreement. Their scheme is more vulnerable to smart card stolen or loss attack, user impersonation and server masquerading attack. Besides, user's identity is traceable in their scheme and their scheme lacks of the forward secrecy. Then, we propose an improved scheme to resist those attacks and analyze the security of our scheme. After that, the advantages of our scheme have been shown by contrast of the performance and efficiency with other three schemes. It shows that our proposed scheme is more secure and applicable to practice.

\section{References}

[1] L. Lamport, "Password authentication with insecure communication", Communications of the ACM, vol. 24, no. 11, (1981), pp. 770-772.

[2] C. C. Chang and T. C. Wu, "Remote password authentication with smartcards", IEE Proceedings-E Computers and Digital Techniques, vol. 138, no. 3, (1993), pp. 165-168.

[3] M. S. Hwang and L. H. Li, "New remote user authentication scheme using smart cards", IEEE Transactions on Consumer Electronics, vol. 46, no. 1,(2000), pp. 28-30.

[4] Y. L. Tang, M. S. Hwang and C. C. Lee, "A simple remote user authentication scheme", Mathematical and Computer Modeling, vol. 36, Issues 1-2, (2002), pp. 103-107.

[5] H. Y. Chien, J. K. Jan and Y. M. Tseng, "An efficient and practical solution to remote authentication: smart card", Computers and Security, vol. 21, no. 4, (2002), pp. 372-375.

[6] C. L. Hsu, "Security of Chien, et al., "Remote user authentication scheme using smart cards", Computer Standards and Interfaces, vol. 26, no. 3 , (2004), pp. 167-169.

[7] W. C. Ku and S. M. Chen, "Weaknesses and improvements of an efficient password based remote user authentication scheme using smart cards", IEEE Transactions on Consumer Electronics, vol. 50, no. 1, (2004), pp. 204-207.

[8] Y. F. Chang and C. C. Chang, "Authentication schemes with no verification Table", Applied Mathematics and Computation, vol. 167, no. 2, (2005), pp. 820-832.

[9] H. C. Hsiang and W. K. Shih, "Weakness and improvements of the Yoon-Ryu-Yoo remote user authentication scheme using smartcards", Computer Communications, vol. 32, no. 4, (2009), pp. 649-652.

[10] M. L. Das, A. Saxena and V. P. Gulati, "A Dynamic ID-based Remote User Authentication Scheme", IEEE Transactions on Consumer 630 Electronics, vol. 50, no. 2, (2004), pp. 639-931.

[11] Y. Y. Wang, J. Y. Liu, F. X. Xiao and J. Dan, "A more efficient and secure dynamic ID-based remote user authentication scheme," Computer Communications, vol. 32, no. 4, (2009), pp. 583-585.

[12] R. Madhusudhan and R. C. Mittal, "Dynamic ID-based remote user password authentication schemes using smart cards: A review", Journal of network and computer applications, vol. 35, no. 4, (2012), pp. 1235-1248.

[13] C. C. Lee, T. H. Lin and R.X. Chang, "A secure dynamic ID based remote user authentication scheme for multi-server environment using smart cards", Expert systems with applications, vol. 38. no. 11, (2011), pp. 13863-13870.

[14] C. G. Ma, D. Wang and S. D. Zhao, "Security flaws in two improved remote user authentication schemes using smart cards", International journal of communication systems, vol. 27, no. 10, (2014), pp. 2215-2227.

[15] L. Xiong, J. W. Niu, Y. Liu, J. Liao and W. Liang, "Robust dynamic ID-based remote user authentication scheme using smart cards", International journal of ad hoc and ubiquitous computing, vol. 17, no. 4, (2014), pp. 254-264.

[16] W. F. Tong, G. D. Li and L. X. Lei, "Cryptanalysis of a New Dynamic ID-based User Authentication Scheme to Resist Smart-Card-Theft Attack", Applied Mathematics \& Information Sciences, vol. 8, no. 4, (2014), pp. 1855-1858.

[17] M. K. Khan, S. Kumari, X. M.Wang and R. Kumar, "Security Issues of Chen et al., dynamic ID-based authentication scheme", 2014 IEEE 12th International Conference on Dependable, Autonomic and Secure Computing (DASC), (2014) August 24-27, Dalian, China.

[18] J. Sand and W. B. Hsieh, "Efficient and secure dynamic ID-based remote user authentication scheme for distributed systems using smart cards", IET information security, vol. 8, no. 2, (2014), pp. 104-113.

[19] X. Xu, Z. P. Jin, H. Zhang and P. Zhu, "A dynamic ID-based authentication scheme based on ECC for telecare medicine Information systems", Applied mechanics and materials, vol. 457-458, (2014), pp. 861-866. 
[20] R. Martinez-Pelaez, F. Rico-Novella, J. Forne and P. Velarde-Alvarado, "Security Improvement of Two Dynamic ID-based Authentication Schemes by Sood-Sarje-Singh", Journal of applied research and technology, vol. 11, (2013), pp. 755-763.

[21] D. Z. Sun and Z. F. Cao, "On the Privacy of Khan et al., Dynamic ID-Based Remote Authentication Scheme with User Anonymity", Cryptologia, vol. 37, no. 4, (2013), pp. 345-355.

[22] Y. F. Chang and H. C. Chang, "Security of dynamic ID-based remote user authentication scheme," 2009 Fifth International Joint Conference on INC, IMS and IDC, (2009), pp. 2108-2110.

[23] Y. F. Chang, W. L. Tai and H. C. Chang, "Untraceable dynamic-identity-based remote user authentication scheme with verifiable password update", International journal of communication systems, vol. 27, no. 11, (2013), pp. 3430-3440.

[24] W. F. Tong and L. X. Lei, "An improved dynamic ID-based remote user authentication with key agreement scheme", Computers \& Electrical engineering, vol. 38, no. 2, (2012), pp. 381-387.

[25] E. J. Yoon and K. Y. Yoo, "On the Security of an Efficient and Secure Dynamic ID-Based Remote User Authentication Scheme", IEICE transaction on information and systems, vol. E95D, no. 6, (2012), pp. 1684-1686.

[26] S. Kumari, M. K. Khan and X. Li, "An improved remote user authentication scheme with key agreement", Computers and Electrical Engineering, vol. 40, no. 6, (2014), pp. 1997-2012.

[27] T. S. Messerges, E. A. Dabbish and R. H. Sloan, "Examining smart-card security under the threat of power analysis attacks", IEEE Trans Comp, vol. 51, no. 5, (2002), pp. 541-52.

[28] P. Kocher, J. Jaffe and B. Jun, "Differential power analysis", Proceedings of advances in cryptology CRYPTO'99, (1999), pp. 388-97.

[29] J. S. Chou, C. H. Huang, Y. S. Huang and Y. Chen, "Efficient two-pass anonymous identity authentication using smart card", IACR Cryptology ePrint Archive, 2013.402th, (2013).

\section{Authors}

Yajuan Shi, is working as a MS candidate in applied mathematics in Wuhan University now. Her research interests include cryptographic protocol ,network security and cryptography.

Han Shen, received his BS degree in basic mathematics from Wuhan University, China, in 2011. After that, he received MS degree in applied mathematics in Wuhan University. Now, he is working as a $\mathrm{PhD}$ candidate in applied mathematics. His research interests include side channel attack and signature scheme.

Yuanyuan Zhang, received her MS degree in applied mathematics from Wuhan University, China, in 2012. Now, she is working as a $\mathrm{PhD}$ candidate in applied mathematics in Wuhan University. Her research interests include cloud computing security and cryptographic protocol.

Jianhua Chen, received his BS degree in applied mathematics from Harbin Institute of Technology, Harbin, China, in 1983 and received his $\mathrm{MS}$ and $\mathrm{PhD}$ degree in applied mathematics from Wuhan University, Wuhan, China, in 1989 and 1994, respectively. Currently, he is a professor of Wuhan University. His current research interests include number theory, information security, and network security. 